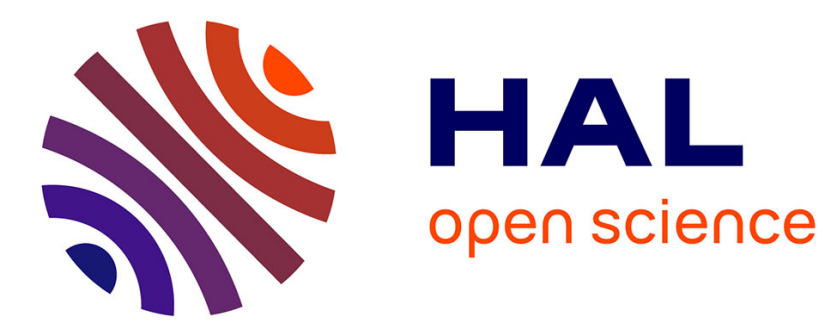

\title{
Étude et réalisation d'amplificateur à transistor à effet de champ a l'AsGa refroidi à très basse température \\ D. Brunet-Brunol
}

\section{To cite this version:}

D. Brunet-Brunol. Étude et réalisation d'amplificateur à transistor à effet de champ a l'AsGa refroidi à très basse température. Revue de Physique Appliquée, 1978, 13 (4), pp.180-187. 10.1051/rphysap:01978001304018000 . jpa-00244438

\section{HAL Id: jpa-00244438 https://hal.science/jpa-00244438}

Submitted on 1 Jan 1978

HAL is a multi-disciplinary open access archive for the deposit and dissemination of scientific research documents, whether they are published or not. The documents may come from teaching and research institutions in France or abroad, or from public or private research centers.
L'archive ouverte pluridisciplinaire HAL, est destinée au dépôt et à la diffusion de documents scientifiques de niveau recherche, publiés ou non, émanant des établissements d'enseignement et de recherche français ou étrangers, des laboratoires publics ou privés. 
Classification

Physics Abstracts

$07.50-07.20$

\title{
ÉTUDE ET RÉALISATION D'AMPLIFICATEUR A TRANSISTOR A EFFET DE CHAMP A L'AsGa REFROIDI A TRÈS BASSE TEMPÉRATURE
}

\author{
D. BRUNET-BRUNOL \\ Institut d'Electronique Fondamentale $(*)$, \\ Université Paris XI, Bât. 220, 91405 Orsay, France
}

(Reçu le 30 septembre 1977, révisé le 15 décembre 1977, accepté le 5 janvier 1978)

\begin{abstract}
Résumé. - On calcule le facteur de bruit minimum et l'impédance de source optimale des transistors à effet de champ à l'AsGa refroidis à très basse température. Cette étude tient compte de l'excès de bruit dans les basses fréquences de la bande micro-onde que l'on ne peut négliger car par exemple à $300 \mathrm{MHz}$, il est plus important que les autres sources de bruit. On étudie l'influence du refroidissement sur les paramètres du transistor à partir des modifications des caractéristiques physiques du matériau semiconducteur.

Des amplificateurs à $300 \mathrm{MHz}$ utilisant des transistors à effet de champ à l'AsGa (NE 244 (1) et Gat $1(2)$ ) ont été réalisés et refroidis dans l'azote liquide et dans l'hélium liquide. Les caractéristiques du point de vue du bruit de ces amplificateurs correspondent aux prévisions théoriques. On a pu obtenir avec un amplificateur refroidi à l'hélium liquide utilisant un transistor NE 244 un facteur de bruit $0,25 \mathrm{~dB}$.

Abstract. - Minimum noise figure and optimum source impedance of GaAs field-effect transistors cooled at cryogenic temperatures are computed. Noise characteristics are derived with excess of noise in low frequencies of the microwave band taken into account ; this low frequency noise nonnegligible at $300 \mathrm{MHz}$ exceeds other sources of noise. Cooling influence was correlated to the physical changes of the material.

Amplifiers using GaAs field-effect transistors (NE 244 and Gat 1) were designed at $300 \mathrm{MHz}$ and cooled in liquide nitrogen and liquid helium. Noise performances of this amplifiers fits well with theory predictions. The liquid helium cooled amplifier using the transistor NE 244 has a noise figure of $0.25 \mathrm{~dB}$.
\end{abstract}

Introduction. - Dans de nombreuses expériences de physique, l'amplification de signaux très faibles nécessite souvent l'emploi de dispositifs refroidis pour diminuer le bruit d'origine thermique, c'est le cas par exemple de certains amplificateurs paramétriques qui sont des systèmes relativement complexes.

L'apparition récente de transistors à effet de champ à l'Arséniure de Gallium offre des possibilités très intéressantes d'amplification à faible bruit à très basse température jusqu'à des fréquences de plusieurs Gigahertz.

Il est donc particulièrement utile de déterminer les paramètres qui interviennent dans le calcul des amplificateurs et de suivre leur évolution en fonction de la température pour optimiser les performances de ce type d'amplificateur.

Les transistors à effet de champ introduits par Shockley [1] en 1952 n'ont pris une place importante

(*) Laboratoire associé au C. N. R. S.

(1) Transistor fourni par NEC.

(2) Transistor fourni par Plessey. dans les applications hyper-fréquences qu'avec l'appa rition du MESFET qui utilise une barrière Schottky à la grille. La grande mobilité du semiconducteur utilisé (l'arséniure de gallium) permet d'obtenir des gains importants, de faibles facteurs de bruit et de hautes fréquences de coupure.

Ce type de transistor constitue de 1 à $18 \mathrm{GHz}$ le dispositif simple le plus performant du point de vue du bruit. De $100 \mathrm{MHz}$ à $1 \mathrm{GHz}$, les performances des MESFET à l'AsGa et des transistors bipolaires sont très proches. Cependant, le refroidissement des MESFET diminue fortement leur bruit, essentiellement d'origine thermique. Cette propriété est particulièrement intéressante lorsqu'il est nécessaire d'amplifier des signaux issus de capteurs fonctionnant à très basse température, tel les SQUID (anneaux supraconducteurs contenant une jonction Josephson) qui permettent des mesures de flux magnétique avec une très grande sensibilité. L'amplificateur réalisé peut ainsi être placé près de la sonde contenant le SQUID au contact de l'hélium liquide et la température de fonctionnement de la partie active du transistor peut être 
optimisée pour obtenir le facteur de bruit le plus faible possible.

On a fabriqué de nombreux amplificateurs à MESFET à l'AsGa mais ils ont été assez rarement conçus pour être refroidis à très basse température : Leitchi $[2,3]$ a réalisé un amplificateur fonctionnant à $12 \mathrm{GHz}$, il l'a refroidi jusqu'à $40 \mathrm{~K}$ et a obtenu un facteur de bruit de 1,8 dB ; Hoult et Richards [4] ont construit un amplificateur fonctionnant à $130 \mathrm{MHz}$ à $77 \mathrm{~K}$ et ont obtenu un facteur de bruit de 0,3 dB.

Nous présentons dans cet article une analyse détaillée permettant de prévoir le facteur de bruit minimum d'un amplificateur refroidi et de déterminer les circuits d'adaptation et de polarisation qui permettent de l'obtenir ; puis nous décrivons un amplificateur fonctionnant à $300 \mathrm{MHz}$ plongé dans l'hélium liquide que nous avons réalisé et nous donnons ses performances.

1. Importance du bruit basse fréquence sur le facteur de bruit minimum et sur ses conditions de réalisation. - 1.1 INVENTAIRE DES DIFFÉRENTS TYPES DE BRUIT. - Nous avons utilisé pour l'étude du bruit l'analyse faite par Pucel, Hans et Statz $[5,6]$ en faisant de plus intervenir le bruit basse fréquence. Ces auteurs ont retenu pour leurs calculs la notion introduite par Grosvalet et al. [7] de courant limité par la saturation de la vitesse des électrons lorsque le champ électrique $E$ atteint une certaine valeur $E_{\text {sat }}$ appelée champ de saturation $\left(3 \times 10^{3} \mathrm{~V} / \mathrm{cm}\right.$ environ pour l'AsGa). Le canal peut alors être séparé en deux régions : une région $I$ voisine de la source où le champ électrique est faible et les porteurs n'ont pas encore atteint la vitesse de saturation et une région II proche $\mathrm{du}$ drain où cette vitesse est atteinte.

Le bruit dans les FET à l'AsGa provient à la fois des sources associées aux résistances parasites et des sources intrinsèques du dispositif, les sources principales de bruits parasites sont la résistance de métallisation de grille $R_{\mathrm{m}}$, et la résistance grille-source (la résistance de contact de drain $R_{\mathrm{dr}}$ génère un bruit qui est négligeable [5] car contrairement aux autres il n'est pas amplifié).

Le bruit intrinsèque comprend le bruit thermique [8] dans la région I augmenté de l'effet d'électrons chauds [9] et de l'effet de propagation à travers la région II, le bruit de diffusion dans la région II à vitesse saturée et un bruit dit basse fréquence. Nous avons en effet mesuré sur l'amplificateur à $300 \mathrm{MHz}$ un bruit que les deux premiers mécanismes ne suffisaient pas à expliquer ; cet excès de bruit dans la partie basse de la bande micro-onde a déjà été remarqué par ailleurs : $[10,11,12]$ il provient de fluctuations de la densité des porteurs dans le canal dues à des centres de générationrecombinaison dans la région de charge d'espace [13, 14].

1.2 CAlCul DU FACTEUR DE BRUIT. - On représente (Fig. 1) comme Pucel et al. [5] les sources de bruit internes du transistor intrinsèque par des géné-

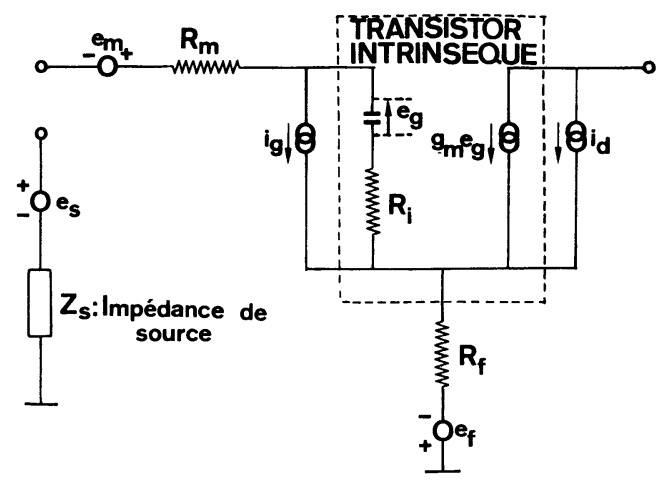

FIG. 1. - Circuit équivalent utilisé dans le calcul du bruit.

[Equivalent circuit for noise analysis.]

rateurs de bruit [8] connectés à l'entrée $\left(i_{\mathrm{g}}\right)$ et à la sortie $\left(i_{\mathrm{d}}\right)$. On y ajoute les sources du bruit dues aux résistances parasites. Le circuit équivalent au transistor intrinsèque utilisé pour l'analyse du bruit est obtenu en négligeant les capacités drain-grille, source-drain et la résistance de drain $[8,15]$.

Les deux composantes du bruit $\left|\bar{i}_{\mathrm{d}}^{2}\right|$ et du bruit $\left|\bar{i}_{\mathrm{g}}^{2}\right|$ dues au bruit thermique et au bruit de diffusion ont été étudiées par Pucel et al. Nous nous intéresseront à leurs composantes $\left|\bar{i}_{\mathrm{dbf}}^{2}\right|$ et $\left|\bar{i}_{\mathrm{gbf}}^{2}\right|$ correspondant au bruit basse fréquence. Le bruit basse fréquence $\left|i_{\mathrm{dbf}}^{2}\right|$ est semblable à celui observé par Van du Ziel [11] sur les JFET ; il est de la forme.

$$
\left|i_{\mathrm{dbf}}^{2}\right|=\frac{k}{1+\omega^{2} \tau^{2}}
$$

où $\tau$ est une constante de temps dépendant des centres de génération-recombinaison; $k$ est une constante du transistor.

A cette composante de $\left|i_{\mathrm{d}}^{2}\right|$ correspond en raison $\mathrm{du}$ couplage capacitif entre le canal et la grille la composante $\left|i_{\mathrm{gbf}}^{2}\right| \mathrm{de}\left|i_{\mathrm{g}}^{2}\right|$ de la forme :

$$
\left|i_{\mathrm{gbf}}^{2}\right|=\frac{k^{\prime} \omega^{2}}{1+\omega^{2} \tau^{2}} .
$$

Pour les fréquences suffisamment hautes $\left(\omega \gg \tau^{1}\right.$ : c'est le cas dans toute la bande micro-onde) cette composante est indépendante de la fréquence, contrairement aux autres qui lui sont proportionnelles.

A partir de la figure 1, Pucel et al. ont mis le facteur de bruit $F$ sous la forme classique :

$$
F=1+\frac{1}{R_{\mathrm{s}}}\left(r_{\mathrm{n}}+g_{\mathrm{n}}\left|Z_{\mathrm{s}}+Z_{\mathrm{c}}\right|^{2}\right)
$$

où $r_{\mathrm{n}}, g_{\mathrm{n}}$ et $Z_{\mathrm{c}}=R_{\mathrm{c}}+j X_{\mathrm{c}}$ sont fonctions des bruits intrinsèques $\left|i_{\mathrm{d}}^{2}\right|$ et $\left|i_{\mathrm{g}}^{2}\right|$, des résistances parasites $R_{\mathrm{m}}$ et $R_{\mathrm{f}}$ et des paramètres petits signaux.

La prise en compte des composantes de $\left|i_{\mathrm{d}}^{2}\right|$ et $\left|i_{\mathrm{g}}^{2}\right|$ correspondant au bruit basse fréquence n'a pas d'influence sur $r_{\mathrm{n}}$ et $\boldsymbol{R}_{\mathrm{c}}$ car les termes correspondant 
aux résistances parasites sont de toute façon prépondérants. Nous avons supposé, pour simplifier, que le bruit bf n'a pas d'influence non plus sur $X_{\mathrm{c}}$; la valeur de $X_{\mathrm{c}}$ n'intervient d'ailleurs pas dans le calcul du facteur de bruit minimum, mais seulement dans celui de l'impédance d'entrée optimale. La prise en compte dans $\left|i_{\mathrm{g}}^{2}\right|$ d'une composante indépendante de la fréquence entraîne en première approximation l'existence dans $g_{\mathrm{n}}$ d'un terme $G$ indépendant de la fréquence. Nous obtenons donc les mêmes expressions finales que Pucel et al. de $r_{\mathrm{n}}, g_{\mathrm{n}}$ et $Z_{\mathrm{c}}$ en fonction des paramètres petits signaux, de la fréquence et de trois paramètres $K_{\mathrm{g}}, K_{\mathrm{c}}$ et $K_{\mathrm{r}}$ (dont ils donnent les courbes en fonction du rapport $L / a$ et de la polarisation) [5] au terme $G$ près :

$$
\begin{aligned}
& r_{\mathrm{n}}=R_{\mathrm{m}}+R_{\mathrm{f}}+K_{\mathrm{r}}\left(\frac{1+\omega^{2} C_{\mathrm{sg}}^{2} R_{\mathrm{i}}^{2}}{g_{\mathrm{m}}}\right) \\
& g_{\mathrm{n}}=K_{\mathrm{g}} \frac{\omega^{2} C_{\mathrm{sg}}^{2}}{g_{\mathrm{m}}}+G \\
& Z_{\mathrm{c}}=R_{\mathrm{m}}+R_{\mathrm{f}}+K_{\mathrm{c}} R_{\mathrm{i}}-j \frac{K_{\mathrm{c}}}{\omega C_{\mathrm{sg}}} .
\end{aligned}
$$

1.3 VARIATION DU FACTEUR DE BRUIT MINIMUM $\boldsymbol{F}_{\min }$ ET DE L'INPÉDANCE D'ENTRÉE OPTIMALE AVEC LA FRÉQUENCE. - Le facteur de bruit minimum $F_{\min }$ est obtenu pour l'impédance d'entrée optimale $R_{\text {sopt }}+j X_{\text {sopt }}$ :

$$
\begin{aligned}
& R_{\text {sopt }}=\left(R_{\mathrm{C}}^{2}+\frac{r_{\mathrm{n}}}{g_{\mathrm{n}}}\right)^{1 / 2} \\
& X_{\text {sopt }}=X_{\mathrm{c}} \\
& F_{\text {min }}=1+2 g_{\mathrm{n}}\left(R_{\mathrm{c}}+R_{\text {sopt }}\right) .
\end{aligned}
$$

Nous avons étudié les variations de ces grandeurs avec la fréquence pour les deux transistors que nous avons utilisés : le Plessey Gat 1 et le NEC NE 244. Nous avons effectué ce calcul pour la polarisation qui donne le bruit le plus faible selon le constructeur et selon nos constatations expérimentales. Tous les paramètres ont été déterminés à partir de la géométrie des transistors et de leur polarisation à l'aide de l'étude de Pucel et al. à l'exception du terme $G$ correspondant au bruit basse fréquence. Ce terme a été évalué en considérant que l'écart constaté vers les basses fréquences (Fig. 2) entre la courbe de $F_{\min }$ en fonction de la fréquence ne tenant pas compte du bruit bf et la courbe donnée par le constructeur [16] lui est dû.

Les courbes de variation de $F_{\min }$ avec la fréquence sont portées sur la figure 2. Elles montrent l'importance du bruit bf. Le facteur de bruit du transistor Gat 1 est plus élevé que celui du transistor NE 244 en raison de résistances parasites plus importantes et d'une capacité $C_{\mathrm{sg}}$ plus importante due à une plus grande longueur de grille. Par contre, le bruit bf est relativement moins élevé que celui du NE 244.

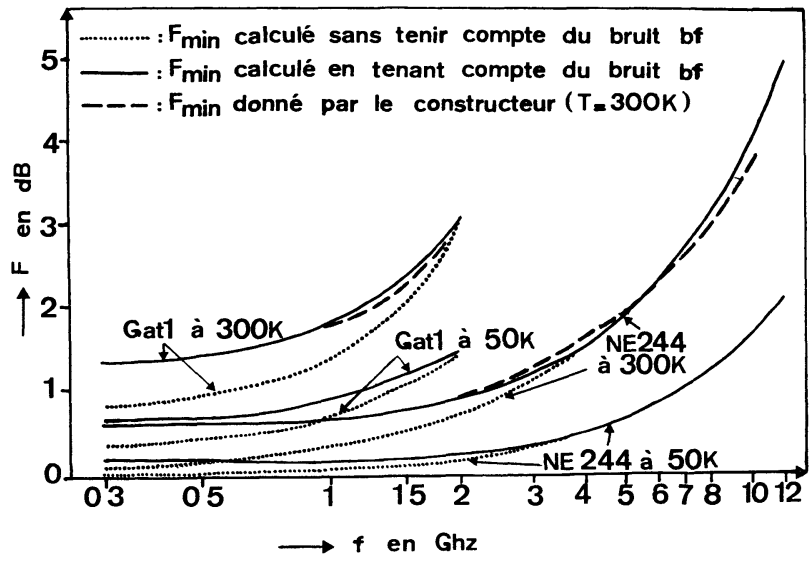

FIG. 2. - Facteur de bruit minimum en fonction de la fréquence à $300 \mathrm{~K}$ et à $50 \mathrm{~K}$.

[Minimum noise figure as a function of frequency at $300 \mathrm{~K}$ and at $50 \mathrm{~K}$.]

L'abaque de Smith de la figure 3 montre les variations de l'impédance d'entrée optimale avec la fréquence pour le transistor NE 244 en tenant compte ou non du bruit bf. Nous avons introduit l'influence des éléments parasites dus au boitier (sensible à partir de $2 \mathrm{GHz}$ en ajoutant directement sur l'abaque les impédances et les admittances correspondantes).
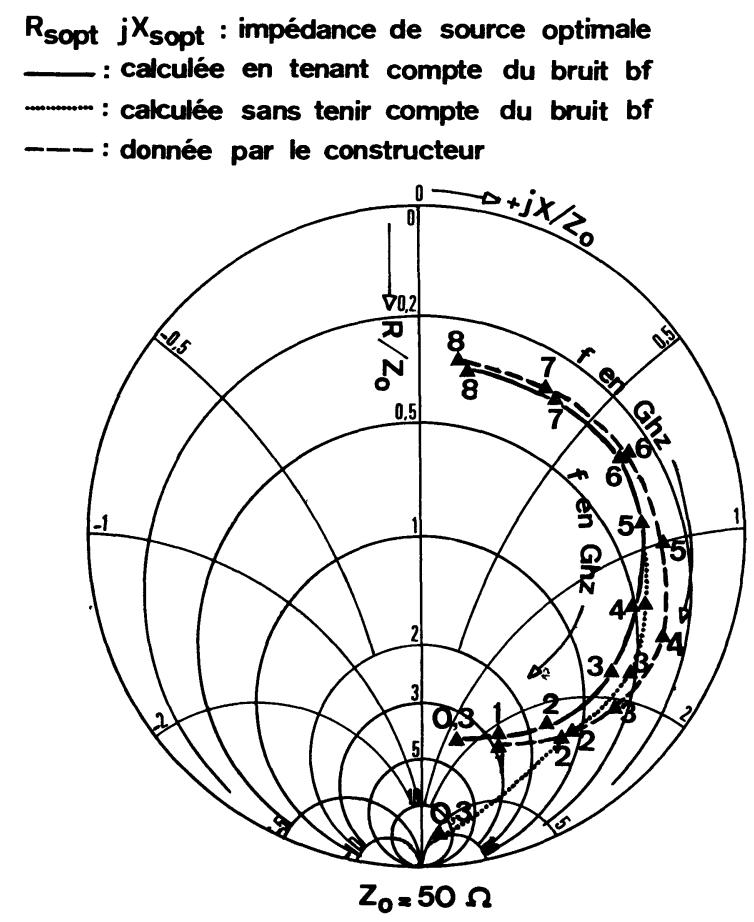

Fig. 3. - Impédance d'entrée optimale en fonction de la fréquence à $300 \mathrm{~K}$.

[Optimum source impedance as a function of frequency at $300 \mathrm{~K}$.

2. Influence du refroidissement à très basse température sur le facteur de bruit minimum et ses conditions de réalisation. -2.1 VARIATION DE LA 
DENSITÉ DES PORTEURS $N$ AVEC LA TEMPÉRATURE. Dans la région d'ionisation des impuretés qui correspond généralement pour l'AsGa à des températures comprises entre 0 et $50 \mathrm{~K}$ lorsqu'il existe un seul type d'impuretés de concentration $N_{\mathrm{D}}$ on peut à partir de la référence [17] calculer la variation de la densité des porteurs $N$ avec la température. Par exemple pour le NE 244 :

$$
N=10^{17} \exp \frac{-23}{T} .
$$

Dans la région d'épuisement des donneurs qui correspond aux températures supérieures à $50 \mathrm{~K}$ :

$$
N=N_{\text {D }} \text {. }
$$

2.2 VARIATION DE LA MOBILITÉ DES PORTEURS $\mu$ AVEC LA TEMPÉRATURE. - A très basse température, les collisions qui prédominent dans le semiconducteur sont dues à la présence d'impuretés ionisées ; la mobilité correspondante $\mu_{\mathrm{I}}$ est donnée dans la référence [18].

A température suffisamment élevée ce sont les collisions dues à l'agitation thermique qui prédominent ; la mobilité correspondante est :

$$
\mu_{\mathrm{R}}=m^{*-5 / 2} T^{-3 / 2}
$$

où $m^{*-5 / 2} T^{-1}$ suivant les références $[19,20]$, la mobilité résultante [21] représentée sur la figure 4 est donc :

$$
\mu=\left(\frac{1}{\mu_{\mathrm{R}}}+\frac{1}{\mu_{\mathrm{I}}}\right)^{-1} .
$$

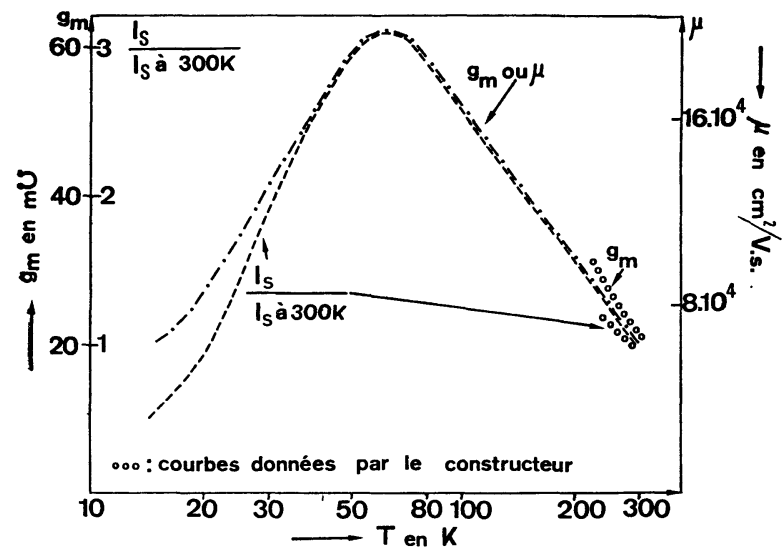

Fig. 4. - Transconductance $g_{\mathrm{m}}$, mobilité des porteurs $\mu$ et courant drain normalisé en fonction de la température.

[Transconductance $g_{\mathrm{m}}$, mobility of carriers $\mu$ and normalized drain current as a function of temperature.]

2.3 VARIATION DU COURANT DRAIN $I_{\mathrm{d}}$ AVEC LA TEMPÉRATURE. - D'après Shockley [1], le courant de saturation de drain $I_{\mathrm{s}}$ est proportionnel à la conducticité du matériau $\sigma=2 q \mu N$. $I_{\mathrm{s}}$ varie donc comme le produit $\mu N$ avec la température. Le courant $I_{\mathrm{d}}$, produit de $I_{\mathrm{s}}$ et d'un terme [5] ne dépendant pas que de la polarisation varie de la même façon. Ces variations sont représentées sur la figure 4.
2.4 VARIATION DU PARAMÈTRE $g_{\mathrm{m}}$ AVEC LA TEMPÉRATURE. $-g_{\mathrm{m}}$ varie comme la mobilité [1] avec la température (Fig. 4). Le maximum de $g_{\mathrm{m}}$ qui correspond approximativement à l'optimum de bruit et de gain se situe aux alentours de $60 \mathrm{~K}$. La concordance avec les courbes données par le constructeur autour de $300 \mathrm{~K}$ (Fig. 4) est bonne.

2.5 VARIATION DU FACTEUR DE BRUIT AVEC LA TEMPÉRATURE. - L'expression (3) de $F$ est intéressante pour l'étude du refroidissement; elle permet de montrer que les propriétés au point de vue du bruit des transistors avec résistances parasites peuvent être représentées par un réseau très simple (Fig. 5) où

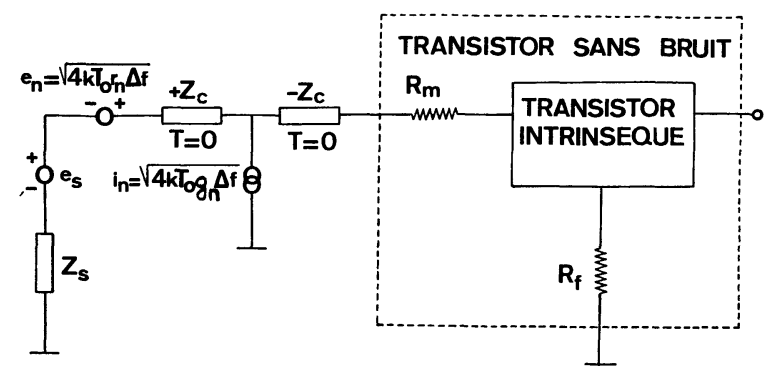

FIG. 5. - Représentation du FET utilisée pour le calcul du bruit de l'amplificateur refroidi.

[FET representation for noise analysis of cooled amplifier.]

toutes les sources de bruit sont ramenées à l'entrée. $r_{n}$ et $g_{n}$ sont les générateurs de tension et de courant de bruit thermique à la température normale $T_{0} \simeq 300 \mathrm{~K} ; Z_{\mathrm{c}}$ est une impédance à la température absolue $T=0 ; R_{\mathrm{f}}$ et $R_{\mathrm{m}}$ interviennent dans l'expression de $r_{\mathrm{n}}$ (c'est alors leurs propriétés parasites sur le bruit qui sont prises en considération) et dans l'expression de $Z_{\mathrm{C}}$ (c'est alors leurs propriétés résistives qui sont prises en considération). En d'autres termes, lorsque l'on refroidit le transistor, il faut multiplier $\boldsymbol{R}_{\mathrm{f}}$ et $R_{\mathrm{m}}$ par $T / T_{0}$ seulement dans l'expression de $r_{\mathrm{n}}$.

Les paramètres $K_{\mathrm{g}}, K_{\mathrm{r}}, K_{\mathrm{c}}$ ne varient pas avec la température pour une polarisation identique (car le rapport $I_{\mathrm{d}} / I_{\mathrm{g}}$ optimum pour le bruit dont ils dépendent reste sensiblement constant) [5].

$r_{\mathrm{n}}$ varie proportionnellement à $T$ comme le bruit thermique des résistances parasites $R_{\mathrm{m}}$ et $R_{\mathrm{f}}$ (la partie de $r_{\mathrm{n}}$ ne venant pas des résistances parasites est négligeable).

Dans l'expression [5], la partie de $g_{\mathrm{n}}$ ne tenant pas compte des résistances parasites est inversement proportionnelle à $g_{\mathrm{n}}$ tracé figure 4 . Des études expérimentales [22] ont été faites sur la variation du bruit bf $\left|i_{\mathrm{dbf}}^{2}\right|$ avec la température, en particulier sur le transistor Gat 1. Ce bruit, donc en première approximation le terme $G$, est divisé par un facteur $\alpha$ de l'ordre de quelques unités $(\alpha \simeq 5)$ lorsque l'on passe de $300 \mathrm{~K}$ à $50 \mathrm{~K}$.

$R_{\mathrm{c}}$ reste constant puisqu'il ne fait intervenir que les propriétés résistives des résistances parasites. 
Les expressions (4), (5), (6) permettent de tracer à différentes fréquences les courbes de $F_{\min }$ en fonction de la température en négligeant le bruit bf (Fig. 6) pour le NE 244.

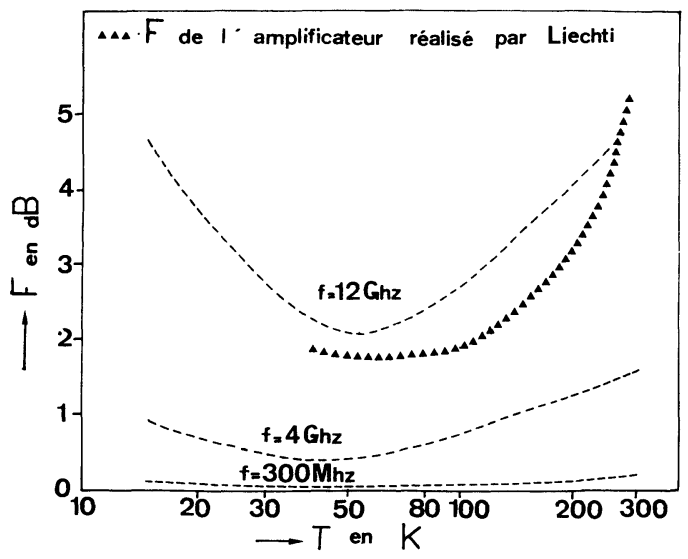

Fig. 6. - Facteur de bruit minimum en fonction de la température pour le transistor NE 244 à différentes fréquences.

[Minimum noise as a function of temperature for transistor NE 244.]

A $12 \mathrm{GHz}$, Liechti [3] a réalisé un amplificateur refroidi et il a mesuré son facteur de bruit (Fig. 6). Le transistor qu'il a utilisé (Hewlett Packard) a des dimensions géométriques comparables à celles du NE 244. Les courbes théoriques de facteur de bruit du NE 244 à $12 \mathrm{GHz}$ et de l'amplificateur refroidi réalisé par Liechti correspondent assez bien ; le refroidissement diminue un peu plus le facteur de bruit de l'amplificateur de Liechti : cette différence peut être due simplement au fait que l'amplificateur adapté du point de vue du bruit à basse température ne l'est pas parfaitement à $300 \mathrm{~K}$.

On peut noter que la température optimale a tendance à diminuer avec la fréquence.

Les courbes de la figure 2 représentent les variations de $F_{\min }$ avec la fréquence pour les transistors NE 244 et Gat 1 à une température ambiante de $50 \mathrm{~K}$ en tenant compte ou non du bruit bf. On constate sur cette figure une amélioration notable due au refroidissement $\left(F_{\min }=0,2 \mathrm{~dB}\right.$ à $50 \mathrm{~K}$ pour le NE 244). Cette amélioration est plus importante si l'on ne tient pas compte du bruit bf qui diminue moins vite avec la température que le bruit thermique. Elle est plus sensible à $300 \mathrm{MHz}$ où le bruit thermique et le bruit bf prédominent qu'à $12 \mathrm{GHz}$ où le bruit de diffusion est important.

2.6 VARIATION DE L'IMPÉDANCE D'ENTRÉE OPTIMUM AVEC LA TEMPÉRATURE. $-X_{\text {sopt }}=-X_{\mathrm{c}}$ ne varie pas lorsque l'on refroidit le transistor.

$$
R_{\mathrm{sopt}}=\left[R_{\mathrm{c}}^{2}+\frac{r_{\mathrm{n}}}{g_{\mathrm{n}}}\right]^{1 / 2}
$$

varie très peu car $R_{\mathrm{c}}$ reste constant, $r_{\mathrm{n}}$ (proportionnel à $T$ ) et $g_{\mathrm{n}}$ (proportionnel à $1 / g_{\mathrm{m}}$ ou à $G$ suivant que le bruit bf est prépondérant ou non, c'est-à-dire suivant la fréquence) varient dans le même ordre de grandeur.

A $12 \mathrm{GHz}$, Liechti avait déjà constaté qu'il n'y avait pas de grosses variations de $Z_{\text {sopt }}$ avec la température. On pourra donc utiliser pour une première approche du circuit d'adaptation l'abaque de la figure 3 .

\section{Réalisation d'un amplificateur FET à l'AsGa} à $300 \mathrm{MHz}$ refroidi. - 3.1 RÉALISATION DE L'ADAPTATION EN BRUIT. - Nous avons défini l'impédance de source optimale du point de vue du bruit; il faut la distinguer de l'impédance qui correspond à une adaptation en puissance : elles peuvent être sensiblement différentes.

3.1.1 Adaptation à l'entrée. $-Z_{\text {sopt }}$ est de l'ordre de quelques centaines d'ohms pour les deux transistors utilisés; pour réaliser une adaptation à $50 \Omega$, nous utilisons un autotransformateur en parallèle avec un condensateur ajustable $C_{1}$ (Fig. 7). Le calcul des éléments du circuit donne :

$$
a^{2}=R_{0} G_{\text {sopt }}
$$

où

$$
\begin{aligned}
& \frac{1}{Z_{\mathrm{sopt}}}=G_{\mathrm{sopt}}+j B_{\mathrm{sopt}} \\
& C_{1} \omega-\frac{1}{L_{1} \omega}=B_{\mathrm{sopt}}
\end{aligned}
$$

$R_{0}=50 \Omega . a$ est le rapport de transformation de l'autotransformateur. $L_{1}$ est son inductance totale.

Le réglage du rapport de transformation $a$ permet d'adapter la conductance ; le réglage du condensateur variable $C_{1}$ permet d'adapter la susceptance.

3.1.2 Adaptation en sortie. - Le circuit d'adaptation en sortie comprend un condensateur ajustable $C_{2}$ en parallèle sur une inductance $L_{2}$, placés en série avec un condensateur ajustable $C_{3}$ (Fig. 7). Pour

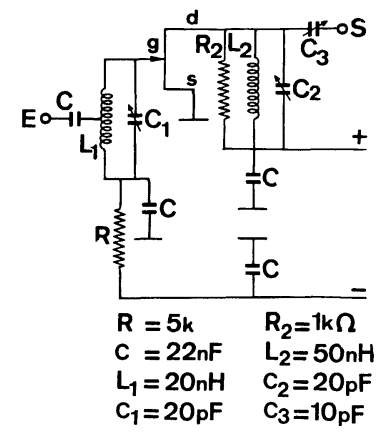

(a)

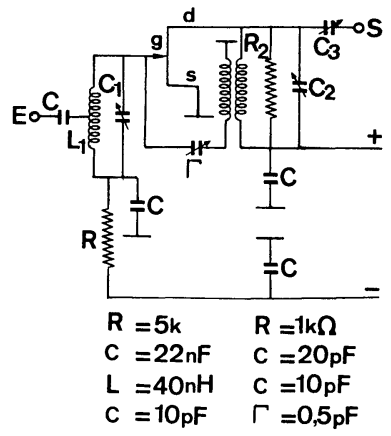

(b)
FIG. 7a. - Amplificateur faible bruit à $300 \mathrm{MHz}$ utilisant le NE 244.

[300 MHz low noise amplifier using NE 244.]

FIG. 7b. - Amplificateur faible bruit à $300 \mathrm{MHz}$ utilisant le Gat 1.

[300 MHz low noise amplifier using Gat 1.] 
avoir en sortie une adaptation en puissance nous devons réaliser une admittance de sortie :

$$
Y=\left[g_{22}-\frac{y_{21} y_{12}}{y_{11}+y_{\text {sopt }}}\right] \text {. }
$$

Le calcul des éléments du circuit d'adaptation $C_{2}$, $L_{2}$ et $C_{3}$ montre qu'ici c'est le réglage du condensateur $C_{3}$ qui permet d'adapter la conductance; ce ne serait pas possible à l'entrée car la résistance du transistor est trop élevée par rapport à $50 \Omega$. Le condensateur $C_{2}$ permet d'ajuster la susceptance, la conductance étant adaptée.

3.2 Stabilité. - 3.2.1 Amplificateur réalisé avec le transistor NE 244. - Nous avons stabilisé l'amplificateur en plaçant une résistance $R_{2}$ suffisamment petite (calculée à partir des paramètres $y$ du transistor) en parallèle à la sortie. $R_{2}$ doit cependant être suffisamment grande pour ne pas augmenter le facteur de bruit ; le courant de bruit dû à $R_{2}$ en sortie doit être négligeable devant le courant de bruit dû à l'amplificateur. Nous avons choisi $R_{2}=1000 \Omega$. Le système est resté stable lorsqu'on l'a refroidi.

3.2.2 Amplificateur réalisé avec le Gat 1. - Nous avons pu stabiliser cet amplificateur, à température normale, par une résistance $R_{2}=500 \Omega$ calculée comme précédemment. Lorsque l'on refroidit ce transistor il est impossible de le rendre inconditionnellement stable par cette méthode, car comme l'ont déjà constaté certains auteurs [23] la capacité grille-drain $C_{\mathrm{gd}}$ augmente à basse température $(0,2 \mathrm{pF}$ à $77 \mathrm{~K}$ contre $0,05 \mathrm{pF}$ à $300 \mathrm{~K}$ ) : il s'établit une forte contreréaction entrée-sortie qui rend le dispositif instable.

Nous avons donc dû neutrodyner la capacité grilledrain ; la self à réaliser pour cela étant trop grosse, le neutrodynage est obtenu à l'aide d'un transformateur inverseur dont le primaire sert d'inductance $L_{2}$ et d'une petite capacité $\Gamma$. Avec un transformateur de rapport de transformation -1 on doit utiliser $\Gamma=C_{\mathrm{dg}}$. Le calcul des paramètres $y$ montre qu'ils varient un peu avec le neutrodynage ; cependant, nous pouvons garder le même circuit et parfaire simplement le réglage des condensateurs $C_{1}, C_{2}, C_{3}$ et du point milieu de l'autotransformateur.

3.3 CirCuItS et RÉGlageS. - L'amplificateur Gat 1 a été réalisé dans un boîtier classique et trempé uniquement dans l'azote liquide. L'amplificateur NE 244 a été réalisé dans un boîtier spécial qui permet de le tremper directement dans l'hélium liquide. Les circuits sont représentés sur la figure 7 .

En raison de leurs faibles dimensions, les FET à l'AsGa peuvent être détruits s'ils sont soumis à de grandes impulsions de tensions transitoires; il faut donc prendre certaines précautions dans le circuit de polarisation: on a réalisé un découplage par une capacité shuntée par une diode Zener et mis une résistance en série avec la grille.

Les éléments des circuits d'adaptation doivent avoir une valeur stable entre $300 \mathrm{~K}$ et $4,2 \mathrm{~K}$; on peut cependant tolérer une légère variation car on doit réajuster à froid ces circuits. Les résistances sont à couches métalliques; leur variation avec la température est inférieure à $1 \%$. Les condensateurs ajustables sont des condensateurs à air.

Les capacités de liaison sont des capacités céramiques dont la valeur initiale est divisée par 3 environ à $4,2 \mathrm{~K}$. La capacité de neutrodynage, très faible est faite de deux petits fils isolés collés l'un à l'autre.

On règle le point milieu de l'autotransformateur et le condensateur $C_{1}$ en connectant à l'entrée à la place $\mathrm{du}$ transistor une admittance $Y_{\text {sopt }}$; on ajuste les éléments jusqu'à ce que le taux d'onde stationnaire à l'entrée soit égal à 1 . On procède de la même manière en sortie. Le réglage du neutrodynage est considéré comme correct quand l'adaptation en sortie reste identique que l'entrée soit fermée ou non sur $50 \Omega$. Ces réglages permettent de réaliser une première adaptation. L'adaptation optimale en bruit est bien plus délicate : elle est faite pas à pas en mesurant le bruit et en modifiant l'adaptation à l'entrée pour l'améliorer. Par commodité, un préréglage de l'amplificateur refroidi est réalisé à la température de l'azote liquide : nous verrons que ce réglage est identique, que l'amplificateur soit plongé dans l'azote liquide ou dans l'hélium liquide.

3.4 Résultats. - Expérimentalement, on constate que les caractéristiques au point de vue courant drain, gain et facteur de bruit de l'amplificateur plongé dans l'azote liquide et de celui plongé dans l'hélium liquide sont pratiquement identiques. Ce résultat est expliqué par Sesnic et al. [24] : le transistor fonctionne à une température nettement supérieure à la température du bain par suite de la puissance dissipée et du mauvais contact thermique avec l'extérieur. Nous avons pu à partir de l'étude de Sesnic et al. [24], de la nature des fils de connexions et de la polarisation utilisée déterminer l'ordre de grandeur de la température réelle : le transistor plongé dans l'hélium liquide fonctionne environ à $50 \mathrm{~K}$, celui plongé dans l'azote liquide à $90 \mathrm{~K}$. Dans les deux cas, nous sommes près du maximum de gain et du minimum de bruit : il est donc logique, qu'il n'y ait pas de différence sensible. On augmente la température de fonctionnement du transistor en augmentant la tension ou le courant de polarisation : on doit donc garder des valeurs de polarisation assez faibles.

Le dispositif de mesure du facteur de bruit utilisé est représenté sur la figure 8 . La charge $50 \Omega$ est soit à température normale $T_{1}=300 \mathrm{~K}$, soit trempée dans l'hélium liquide $\left(T_{2}=4,2 \mathrm{~K}\right)$. On peut négliger les pertes du mélangeur en raison du gain important des amplificateurs qui le précèdent. La température de bruit mesurée est :

$$
T_{\mathrm{b}}=\frac{V_{2}^{2} T_{1}-V_{1}^{2} T_{2}}{V_{1}^{2}-V_{2}^{2}}
$$




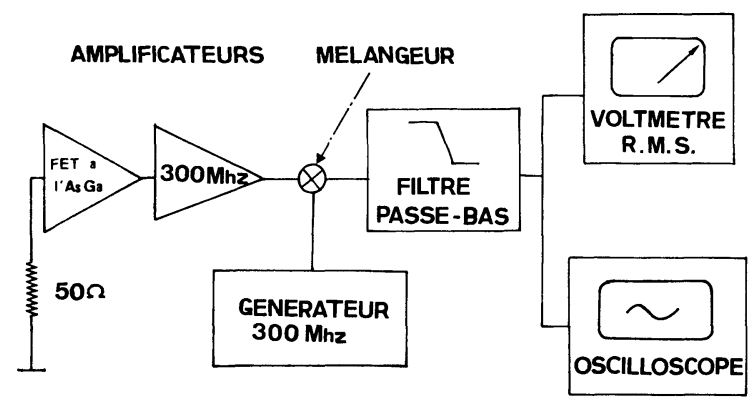

Fig. 8. - Système de mesure du facteur de bruit.

[Noise figure measurement system.]

où $V_{1}^{2}$ et $V_{2}^{2}$ sont les bruits mesurés respectivement en sortie lorsque la charge est à température normale et trempée dans l'hélium liquide.

Pour l'amplificateur utilisant le NE 244 trempé dans l'hélium liquide et parfaitement adapté, le plus performant que nous ayons réalisé on trouve : $F=1,06 \pm 0,02$ soit en décibel $F=0,25 \mathrm{~dB} \pm 0,1 \mathrm{~dB}$. L'incertitude relative assez importante est très difficile à limiter, toutes ses composantes (incertitude due au voltmètre, variation de la résistance avec la température, incertitude sur le calcul de bruit des amplificateurs suivants) étant du même ordre de grandeur.

La figure 9 montre les résultats obtenus à différentes températures. On constate pour les deux tran-

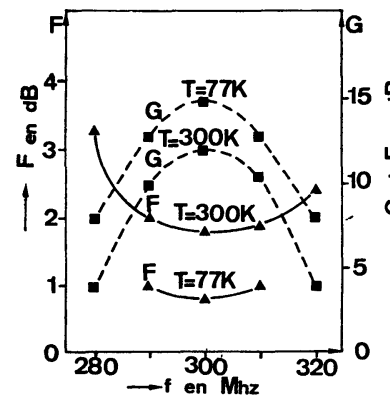

(a)

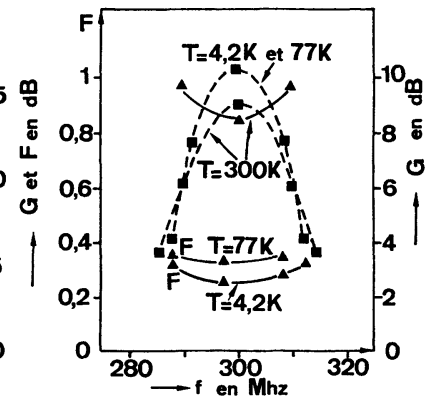

(b)
FIG. 9a. - Facteur de bruit et gain de l'amplificateur utilisant le Gat 1 mesurés.

[Mesured noise figure and gain of the amplifier using Gat 1.]

Fig. 9b. - Facteur de bruit et gain de l'amplificateur utilisant le NE 244 mesurés.

[Measured noise figure and gain of the amplifier using NE 244.]

sistors que le gain augmente lorsque la température diminue. Cette augmentation est moins importante que ne le laissait penser l'accroissement de $g_{\mathrm{m}}$ car ce gain n'est pas le gain maximum mais le gain pour une adaptation en bruit optimale. Ce n'est d'ailleurs pas le même amplificateur qui fonctionne à $4,2 \mathrm{~K}$ et à $300 \mathrm{~K}$, ce sont deux amplificateurs adaptés différemment pour obtenir un bruit minimal. Par contre, c'est le même amplificateur qui fonctionne à $4,2 \mathrm{~K}$ et à $77 \mathrm{~K}$. On constate qu'entre ces deux températures les performances de l'amplificateur changent peu : elles s'améliorent légèrement dans l'hélium où le transistor fonctionne à peu près à la température optimale pour le bruit (environ $50 \mathrm{~K}$ ).

Les facteurs de bruits atteints sont très légèrement supérieurs à ceux que l'on peut espérer d'après les calculs théoriques ${ }_{\mathrm{r}}$ Le refroidissement permet de diminuer le facteur de bruit dans les proportions prévues.

La figure 10 représente les variations du facteur de bruit de l'amplificateur refroidi utilisant le NE 244 avec la polarisation.

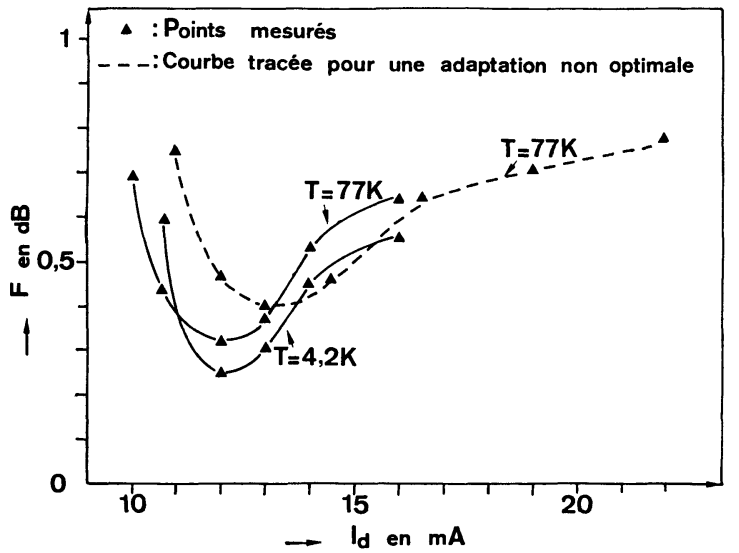

Fig. 10. - Facteur de bruit de l'amplificateur utilisant le NE 244 mesuré en fonction du courant drain.

[Measured noise figure of the amplifier using NE 244 as a function of drain current.]

Cette courbe a bien la forme de celle calculée par Pucel et al. [5] et de celle trouvée expérimentalement par Brehm et Vendelin [25]. Le courant optimum mesuré à température ambiante est $10 \mathrm{~mA}$ comme prévu, mais il n'augmente pas comme prévu quand la température diminue. Une courbe faite pour une adaptation non optimale (le point milieu de l'autotransformateur étant légèrement décalé) montre que ce courant optimum varie légèrement avec l'adaptation.

4. Conclusion. - Nous avons réalisé un amplificateur ayant $0,25 \mathrm{~dB}$ de facteur de bruit à $300 \mathrm{MHz}$ lorsqu'il est plongé dans l'hélium liquide : il fonctionne environ à $50 \mathrm{~K}$. Nous avons montré l'importance du bruit basse fréquence sur ce facteur de bruit. Le refroidissement améliore très sensiblement les performances du transistor. 


\section{Bibliographie}

[1] Shockley, W., Proc. IRE 40 (1952) 1365-1376.

[2] Liechti, A. C., Tillman, L. R., IEEE Trans. MTT-22 (1974) $510-517$.

[3] Liechti, A. C., Larrick, B. R., Mellor, J. D., rapport interne Hewlett Packard.

[4] Hoult, D. I., Richards R. E., Rapport interne Oxford.

[5] Pucel, R. A., Hermann, A. H., Statz, H., Signal and noise properties of GaAs microwave FET.

[6] Statz, H., Hermann, A. H., Pucel, R. A., IEEE Trans. ED-21 (1974) 549-562.

[7] Grosvalet, J., Motsch, C., Tribes, R., Solid States Electron. 6 (1973) 65-67.

[8] VAN DER Ziel, Proc. IEEE 51 (1963) 461-467.

[9] BAEChTOLD, W., IEEE, Trans. ED-18 (1971) 97-104.

[10] BaEchtold, W., DactWryler, K., Forster, T., MoH, T. O., Walter, W., Wolf, P., Electron. Lett. 9 (1973) 232-234.

[11] VAN DER ZIEL, Proc. IEEE 51 (1963) 1670-1671.

[12] Halladay, H. E., Bruncke, W. C., Proc. IEEE 51 (1963) 1671.
[13] LaUritzen, P. O., Solid States Electron. 8 (1965) 41-58.

[14] SAH, C. T., Proc. IEEE 52 (1964) 795-814.

[15] Klaasen, F. M., Prins, J., IEEE Trans ED-16 (1969) 952-952.

[16] Notes techniques du constructeur NEC.

[17] Vapaille, Physique des dispositifs semiconducteurs (ed. Masson) 1970.

[18] Liechti, C., Proc. of 1974 European Microwave Conference, Montreux B 2, p. 87-90.

[19] BeCKTel, N. G., Microwave J. 11 (1972) 15-19.

[20] Sze, Physics of semiconductors Devices (ed. Wiley).

[21] Thèse Oliva, Orsay 1975.

[22] Soldini, D., Toboul, A., Leroy, G., Savelli, M., Electron. Lett. 12 (1976) 42.

[23] Leost, J. C., LoRIou, B., Onde Electrique (1974) 514-521.

[24] Sesnic, S. S., Graig, R. G., IEEE Trans ED-19 (1972) 933-942.

[25] Brehm, G. E., Vendelin, G. D., Microwaves (1974) 38. 